Milva Maria Figueiredo de Martino ${ }^{1}$ Claudia Aparecida Rosa da Silva ${ }^{2}$

Symone Antunes Miguez ${ }^{3}$

\title{
Estudo do cronótipo de um grupo de trabalhadores em turnos
}

\section{Study of a group of shift workers' chronotype}

${ }^{1}$ Professora Doutora Associada do Departamento de Enfermagem da Faculdade de Ciências Médicas da Universidade Estadual de Campinas.

${ }^{2}$ Mestre em Enfermagem na Área de Concentração Enfermagem e Trabalho pelo Departamento de Enfermagem da Faculdade de Ciências Médicas da Universidade Estadual de Campinas. Enfermeira do Serviço de Enfermagem em Obstetrícia da Divisão de Enfermagem do Centro de Atenção Integral à Saúde da Mulher da Universidade Estadual de Campinas. ${ }^{3}$ Mestre em Enfermagem na Área de Concentração Enfermagem e Trabalho pelo Departamento de Enfermagem da Faculdade de Ciências Médicas da Universidade Estadual de Campinas. Fisioterapeuta assessora da Ergocamp Comércio e Assessoria em Ergonomia.

\begin{abstract}
Resumo
Neste estudo, os objetivos foram descrever as características de matutinidade/ vespertinidade de um grupo de trabalhadores em turnos de uma indústria química e identificar possíveis associações entre cronótipo e turno de trabalho.

A pesquisa foi realizada em uma indústria química do interior do estado de São Paulo. Os trabalhadores lotados nesta seção eram distribuídos em dois turnos: matutino e vespertino. A amostra constituiu-se de 44 trabalhadores, dos sexos feminino e masculino, que trabalhavam em ambos os turnos. Os dados foram coletados com a versão brasileira do questionário de identificação de indivíduos matutinos e vespertinos que foi elaborado por Horne \& Östberg (1976).

Foi verificado o predomínio do cronótipo "moderadamente matutino" - MM (n = 21 ou 47,73\%) -, seguido pelo tipo "Indiferente" - IN ( $\mathrm{n}=12$ ou 27,27\%) - e pelo tipo "definitivamente matutino" - DM (n = 10 ou 22,73\%). Somente um indivíduo foi classificado como "definitivamente vespertino" - DV (2,27\%).

Entre os trabalhadores estudados houve predomínio do cronótipo "moderadamente matutino" seguido do tipo "indiferente" e do tipo "definitivamente matutino". Não foram verificadas associações estatisticamente significativas entre o cronótipo e as características individuais neste estudo.
\end{abstract}

Palavras-chave: cronobiologia, cronótipo, turno de trabalho.

\begin{abstract}
This study aimed not only at describing the typical morning or night features of a group of shift workers in a chemical industry, but also at identifying possible links between work shifts and chronotype.

This study was held in a two-shift-chemical industry located in the interior of São Paulo. The subjects of our research were the 44 men and women working there either in the morning or at night. The data was collected through the Brazilian version of the Horne \& Östberg chronotypes identification questionnaire .

The "moderately morning" chronotype was predominant - MM (47,73\%), followed by the "indifferent" - IN (27,27\%) and by the "definitely morning" - DM (22,73\%). Only one of them could be considered a "definitely night" - DN $(2,27 \%)$.

The workers under study were predominantly classified as "moderately morning" followed by the "indifferent" and by the "definitely morning" chronotypes. No statistically relevant relationship was found between the chronotype and individual characteristics.
\end{abstract}

Keywords: chronotype, shift workers, chronobiology. 


\section{Introdução}

Observa-se hoje um contingente enorme de pessoas trabalhando em sistema de turnos, realizando seu trabalho em horários constantes, porém incomum (Rutenfranz, Knauth \& Fisher, 1989). A estruturação do trabalho em sistema de turnos deixou de ser prioridade dos serviços como saúde e segurança, principalmente nos países industrializados, e inúmeros outros serviços passaram a manter a continuidade de sua produção durante as 24 horas do dia, o ano todo.

O trabalho realizado fora dos horários usuais (das $8 \mathrm{~h}$ ou $9 \mathrm{~h}$ até às $17 \mathrm{~h}$ ou $18 \mathrm{~h}$ ) faz parte do grupo de fatores psicossociais que interagem nos processos saúde-doença (Fisher, 2003). Inúmeros estudos demonstraram que essa forma de organização do trabalho pode gerar impactos sobre a saúde e a qualidade de vida dos trabalhadores. Neste estudo, interessanos como a organização do trabalho em sistema de turnos pode interferir na ritmicidade biológica e o enfoque que daremos será a partir da Cronobiologia.

A Cronobiologia, estudo sistemático da organização temporal da matéria viva (MennaBarreto \& Fortunato, 1988), a ciência dos ritmos biológicos, mostra que os organismos vivos são, fisiológica e comportamentalmente, diferentes, dependendo da hora do dia em que são observados, assim como reagem diferentemente a um mesmo estímulo quando aplicado em diferentes momentos das 24 horas do dia (Cipolla-Neto, 1988).

Do interesse de estudar as conseqüências do trabalho em turnos sobre a ritmicidade biológica, enfocamos este estudo na adaptação ou não dos trabalhadores aos turnos de trabalho.

Na adaptação ao trabalho em turnos, um importante fator interno é a preferência em relação aos horários de dormir e despertar do indivíduo, ou seja, o cronótipo ou tipo cronobiológico do indivíduo. A população humana pode ser dividida em três tipos básicos: os indivíduos matutinos, os

\section{Casuística e Métodos}

\section{Local e regime de trabalho}

A pesquisa foi realizada em uma indústria química do interior do estado de São Paulo, no setor de Produtos de Consumo. Os trabalhadores lotados nesta seção eram distribuídos em dois turnos: matutino e vespertino. O regime de trabalho no local é de jornadas de oito horas diárias, sendo das $6 \mathrm{~h}$ às $14 \mathrm{~h}$ no turno matutino e das $14 \mathrm{~h}$ às $22 \mathrm{~h}$ no turno vespertino. vespertinos e os indiferentes. Os indivíduos matutinos despertam espontaneamente muito cedo, por volta das cinco às sete horas da manhã, estando aptos para o trabalho e em um nível de alerta muito bom. Possuem preferência para dormir cedo, constituindo $10 \%$ a $12 \%$ da população. Os indivíduos vespertinos despertam tarde, entre $12 \mathrm{~h}$ e $13 \mathrm{~h}$ quando em férias ou em finais de semana, e dormem tarde, por volta de $2 \mathrm{~h}$ a $3 \mathrm{~h}$ da madrugada, e em dias normais, seu melhor desempenho se dá à tarde ou à noite. Esse tipo constitui $8 \%$ a $10 \%$ da população. O terceiro tipo é dos indivíduos indiferentes, pois não possuem preferência pelo horário de levantarse (Horne \& Östberg, 1976).

O estudo do cronótipo do indivíduo é importante na determinação dos períodos de melhor desempenho e maior bem-estar. Sua identificação pode ser utilizada para otimizar a qualidade do trabalho e minimizar distúrbios associados a este.

Em um estudo analisando os cronótipos de um grupo de enfermeiros docentes de um colégio técnico em um hospital de ensino, os resultados demonstraram a existência de associações entre a preferência do período para a realização do trabalho e o cronótipo dos indivíduos estudados (Campos \& De Martino, 2001).

Um outro estudo, analisando o cronótipo dos auxiliares de enfermagem de um hospital do estado do Paraná, observou-se que nem sempre as características individuais são respeitadas na distribuição dos trabalhadores nos diferentes turnos (Zubioli, Muranda \& Sant'ana, 1998).

Portanto, neste estudo tivemos como objetivos descrever as características de matutinidade e vespertinidade de um grupo de trabalhadores em turnos de uma indústria química e identificar possíveis associações entre cronótipo e turno de trabalho.

\section{Amostra}

A amostra constituiu-se de 44 trabalhadores dos sexos feminino e masculino, tanto do turno matutino quanto do vespertino de uma indústria química do interior do estado de São Paulo.

\section{Instrumento de coleta de dados}

O instrumento utilizado foi o questionário de identificação de indivíduos matutinos e 
vespertinos, que foi elaborado por Horne e Östberg (1976), tendo sido traduzido para a língua portuguesa e aplicado no Brasil após ter sido validado e adaptado às características e hábitos de nossa população por BeneditoSilva et al. 1990. (Anexo 1).

O questionário é composto de questões a respeito de situações habituais da vida diária, e o indivíduo pesquisado deve registrar os seus horários preferenciais em relação à sua vida cotidiana, considerando-se que ele fosse completamente livre para escolher estes horários. O resultado é dado por um valor numérico, que varia entre 16 e 86 pontos, classificando o indivíduo em cinco tipos diferentes: "vespertino extremo" (16 a 30 pontos), "moderadamente vespertino" (31 a 41 pontos), "indiferente" (42 a 58 pontos), "moderadamente matutino" (59 a 69 pontos) e "matutino extremo" (70 a 86 pontos).

\section{Procedimentos de coleta de dados}

Os dados foram coletados pelas pesquisadoras. Os trabalhadores foram abordados em seus locais de trabalho, receberam as informações sobre a pesquisa e relataram se tinham interesse e disponibilidade para participarem da pesquisa. Após o Termo de Consentimento Livre e Esclarecido ter sido assinado, foram entregues os questionários, as pesquisadoras permaneceram no local para solucionar eventuais dúvidas e aguardar a devolução dos mesmos. O período de coleta de dados foi de outubro a novembro de 2003.

\section{Tratamento estatístico}

Foram utilizadas tabelas de freqüências para as variáveis categóricas (Sexo, Turno e Cronótipo) e estatísticas descritivas para as variáveis contínuas (Idade e Tempo na função).

Para verificar se existe associação entre o cronótipo e as variáveis categóricas, foi utilizado o teste Qui-quadrado. Para compararmos as variáveis contínuas com relação ao cronótipo, utilizamos o teste nãoparamétrico de Kruskal-Wallis. Ambos os testes dizem que existe diferença significativa quando o p-valor do teste aplicado é $£ 0,05$.

\section{Resultados}

A amostra em relação ao turno de trabalho constituiu-se de 24 trabalhadores (54,55\%) do turno matutino e $20(45,45 \%)$ do turno vespertino. Nenhum trabalhador era do turno noturno. Dos 44 trabalhadores da

amostra, 27 eram do sexo masculino $(61,36 \%)$ e 17 do sexo feminino $(38,64 \%)$. As estatísticas descritivas para idade e tempo na função (meses) dos trabalhadores estão descritas na tabela 1.

Tabela 1 Estatísticas descritivas das variáveis idade etempo na função (meses) entre trabalhadores de uma indústria química do interior do estado de São Paulo, 2003

\begin{tabular}{lllcccc}
\hline Variável & $\mathrm{N}$ & Média & D.P. & Mínimo & Mediana & Máximo \\
\hline $\begin{array}{r}\text { Idade (anos) } \\
\begin{array}{r}\text { Tempo na função } \\
\text { (meses) }\end{array}\end{array}$ & 44 & 32,36 & 7,74 & 20 & 32,50 & 53 \\
\hline
\end{tabular}

Verificamos que a média de idade dos trabalhadores foi de 32,36 anos e o tempo na função foi de 54,35 meses, independentemente do turno de trabalho.

Através da figura 1, verificamos o predomínio do cronótipo "moderadamente
matutino"-MM (n = 21 ou 47,73\%) -, seguido pelo tipo "Indiferente" - IN (n = 12 ou $27,27 \%$ ) - e pelo tipo "definitivamente matutino" - DM ( $\mathrm{n}=10$ indivíduos ou $22,73 \%)$. Somente um trabalhador foi classificado como "definitivamente vespertino” - DV $(2,27 \%)$.

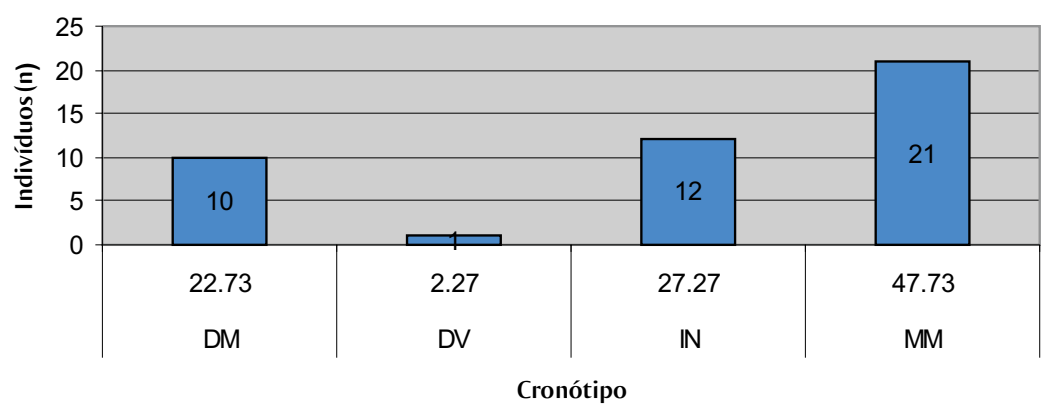

Figura 1 Distribuição dos trabalhadores segundo cronótipo 
O teste do Qui-quadrado foi utilizado para verificar possível associação dos trabalhadores dos turnos matutino e vespertino e seu respectivo cronótipo.

De acordo com a tabela 2, verificamos que 11 trabalhadores do turno matutino $(25,58 \%)$ apresentaram cronótipo "moderadamente matutino", seguidos de 8 trabalhadores $(18,60 \%)$ do tipo "definitivamente matutino" e 5 trabalhadores $(11,63 \%)$ do tipo "indiferente". No turno vespertino, observamos
10 trabalhadores $(23,26 \%)$ do tipo "moderadamente matutino", seguidos de 7 trabalhadores $(16,28 \%)$ do tipo "indiferente" e apenas 2 trabalhadores deste turno (4,65\%) do tipo "definitivamente matutino". Não houve diferença significativa entre cronótipo e turno de trabalho ( $\mathrm{p}$-val=0,1785). Na análise, foi excluído o trabalhador classificado como "definitivamente vespertino" por se tratar de apenas um indivíduo e por constituir, segundo a literatura, um cronótipos mais raro (Figura 2).

Tabela 2 Distribuição segundo cronótipo e turno de trabalho, entre trabalhadores de uma indústria química do interior do estado de São Paulo, 2003

\begin{tabular}{lrrrrrr}
\hline Cronótipo & \multicolumn{2}{c}{ Matutino } & \multicolumn{2}{c}{ Vespertino } & \multicolumn{2}{c}{ Total } \\
& $\mathrm{n}$ & $\%$ & $\mathrm{n}$ & $\%$ & $\mathrm{n}$ & $\%$ \\
\hline DM & 8 & 18,60 & 2 & 4,65 & 10 & 23,26 \\
I & 5 & 11,63 & 7 & 16,28 & 12 & 27,91 \\
MM & 11 & 25,58 & 10 & 23,26 & 21 & 48,84 \\
\hline
\end{tabular}

Teste $x^{2} p$-valor $=0,1785(p=0,05)$

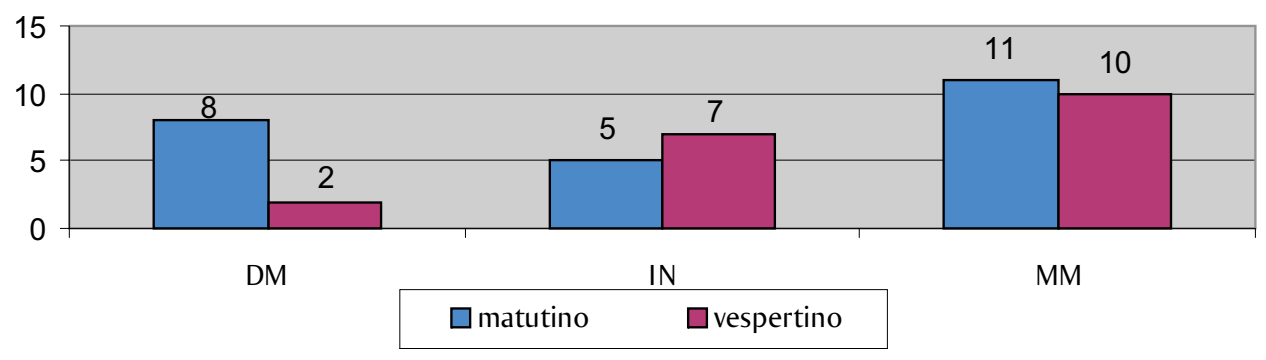

Figura 2 Distribuição dos trabalhadores segundo cronótipo e turnos de trabalho

O teste exato de Fisher foi utilizado para verificar possível associação entre sexo e turno de trabalho.

De acordo com a tabela 3, verificamos que, entre os trabalhadores do sexo feminino (18,60\%), 8 apresentavam cronótipo "moderadamente matutino", seguidos de 5 trabalhadores $(11,63 \%)$ do tipo "definitivamente matutino" e 4 trabalhadores $(9,30 \%)$ do tipo "indiferente".
Observamos que, entre os trabalhadores do sexo masculino $(30,23 \%), 13$ apresentavam cronótipo "moderadamente matutino", seguidos de 8 trabalhadores $(18,60 \%)$ do tipo "indiferente" e 5 trabalhadores (11,63\%) do tipo "definitivamente matutino". Não houve diferença significativa entre sexo e cronótipo (p-val = 0,7808). Nesta análise também foi excluído o trabalhador classificado como “definitivamente vespertino”. (Figura 3)

Tabela 3 Distribuição segundo cronótipo e sexo entre trabalhadores de uma indústria química do interior do estado de São Paulo, 2003

\begin{tabular}{lrrrrrr}
\hline Cronótipo & \multicolumn{2}{c}{ Feminino } & \multicolumn{2}{c}{ Masculino } & \multicolumn{2}{c}{ Total } \\
& $\mathrm{n}$ & $\%$ & $\mathrm{n}$ & $\%$ & $\mathrm{n}$ & $\%$ \\
\hline DM & 5 & 11,63 & 5 & 11,63 & 10 & 23,26 \\
I & 4 & 9,30 & 8 & 18,60 & 12 & 27,91 \\
MM & 8 & 18,60 & 13 & 30,23 & 21 & 48,84 \\
\hline Teste Exato de Fisher p-valor $=0,7808(\mathrm{p}=0,05)$ & & & &
\end{tabular}




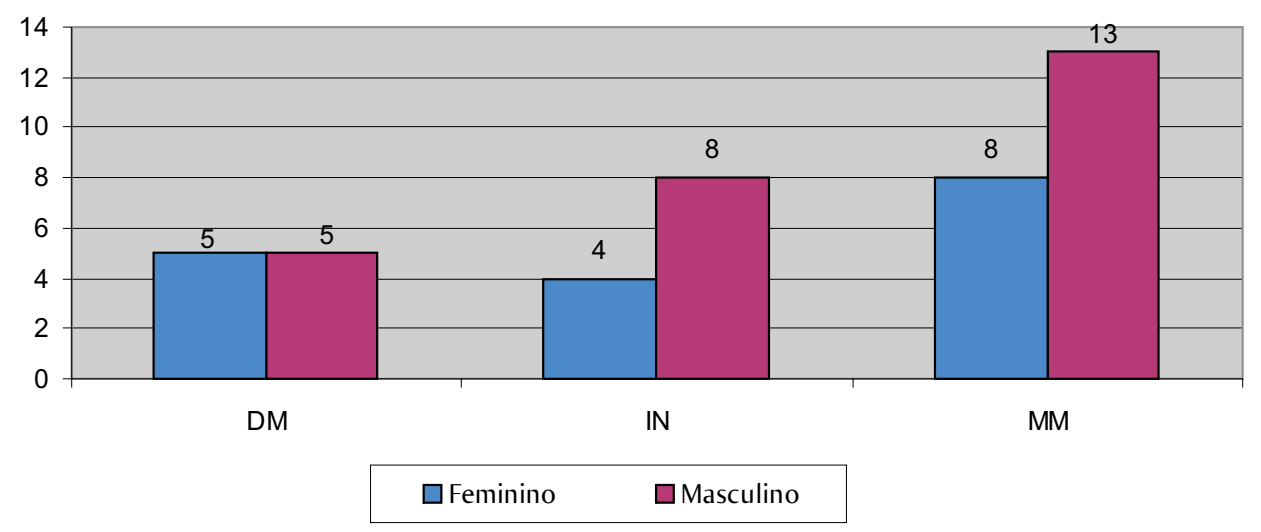

Figura 3 Distribuição dos trabalhadores segundo cronótipo e sexo

As estatísticas descritivas para idade dos trabalhadores, segundo o tipo cronobiológico, estão descritas na tabela 4.

Tabela 4 Estatísticas descritivas da variável Idade, por cronótipo, entre trabalhadores de uma indústria química do interior do estado de São Paulo, 2003

\begin{tabular}{llllccc}
\hline Cronótipo & N & Média & D.P. & Mínimo & Mediana & Máximo \\
\hline DM & 10 & 35,40 & 6,35 & 26 & 35 & 46 \\
I & 12 & 29,25 & 6,36 & 20 & 31,50 & 38 \\
MM & 21 & 33,05 & 8,66 & 22 & 31 & 53 \\
\hline
\end{tabular}

Teste de Kruskal-Wallis $p$-valor $=0.1694(p=0,05)$

Verificamos que a média de idade dos trabalhadores de tipo cronobiológico "moderadamente matutino" foi de 33,05 anos, do tipo "indiferente" foi de 29,25 anos e do tipo "definitivamente matutino" foi de 35,40 anos.
Não houve diferença significativa entre idade e cronótipo ( $\mathrm{p}$-val = 0,1694).

As estatísticas descritivas para tempo na função dos trabalhadores, segundo o tipo cronobiológico, estão descritas na tabela 5.

Tabela 5 Estatísticas descritivas da variável Tempo na Função (meses), por cronótipo, entre trabalhadores de uma indústria química do interior do estado de São Paulo, 2003

\begin{tabular}{llllrrc}
\hline Cronótipo & $\mathrm{N}$ & Média & D.P. & Mínimo & Mediana & Máximo \\
\hline DM & 10 & 48.50 & 47.11 & 7.00 & 30.00 & 144.00 \\
I & 12 & 63.67 & 45.57 & 7.00 & 72.00 & 144.00 \\
MM & 21 & 50.95 & 77.05 & 7.00 & 24.00 & 312.00 \\
\hline
\end{tabular}

Teste de Kruskal-Wallis p-valor $=0.4211(p=0,05)$

Verificamos que o tempo na função dos trabalhadores de tipo cronobiológico "moderadamente matutino" foi de 50,95 meses, do tipo "indiferente" foi de 63,67

\section{Discussão}

Em nossa amostra, tivemos uma distribuição homogênea com relação ao número de trabalhadores por turno de trabalho, o predomínio de trabalhadores do sexo masculino e o tempo na função em média de 4 anos e 6 meses. meses e do tipo "definitivamente matutino" foi de 48,50 meses. Não houve diferença significativa entre tempo na função e cronótipo ( $p$-val = 0,4211).

Segundo a literatura, o cronótipo representa um fator importante para a adaptação dos indivíduos aos turnos de trabalho. Estudos realizados por De Martino \& Ceolim (2001) com um grupo de enfermeiros em um hospital de ensino e por 
Campos \& De Martino (2001) com docentes de enfermagem em um colégio técnico apontaram que as preferências do período para a realização do trabalho foram ao encontro do cronótipo dos envolvidos.

Entre os trabalhadores estudados, houve predomínio do cronótipo "moderadamente matutino" seguido do tipo "indiferente" e do tipo "definitivamente matutino". Em nossa pesquisa, encontramos apenas um trabalhador "definitivamente vespertino", confirmando os dados da literatura de que os indivíduos de cronótipos extremos são mais raros (Horne \& Ostberg, 1976).

A análise dos resultados mostra que houve uma concordância entre o cronótipo e os turnos de trabalho em consonância com os achados de Horne \& Ostberg (1976). Observamos que a maioria dos trabalhadores do turno matutino $(25,58 \%)$ foi classificada como "moderadamente matutino" e 18,60\% como "definitivamente matutino", demonstrando uma associação entre o cronótipo e o turno de trabalho.

Com relação aos cronótipos dos trabalhadores do turno vespertino, encontramos a maioria dos trabalhadores do tipo cronobiológico "moderadamente matutino" $(23,26 \%)$, o que pode acarretar um problema para estes trabalhadores. Estabelecendo-se uma associação entre as características biológicas individuais e as atividades laborativas dos indivíduos, os que se caracterizam ou se definem como vespertinos pela maior disposição para atividades físicas e mentais no período vespertino e noturno seriam os mais indicados para o trabalho no turno vespertino. Segundo Ferreira (1988), levar em consideração as diferenças interindividuais, como a idade e a estrutura circadiana (tipos matutinos e vespertinos), denominada cronótipo, parece ser um fator importante à adaptação das pessoas ao trabalho em turnos.

Verificamos a presença de 11,63\% dos trabalhadores do matutino e $16,28 \%$ dos trabalhadores do vespertino com cronótipo "indiferente". Segundo Rutenfranz, Knauth e Fischer (1989) as pessoas com tipo cronobiológico indiferente se adaptam a qualquer horário.

Segundo a literatura, os indivíduos classificados como "indiferentes" correspondem à imensa maioria das pessoas (Reimão, 1988). Em nossa amostra, houve predomínio do cronótipo "moderadamente matutino", tanto no turno matutino quanto no turno vespertino.
Em relação às variáveis sexo e tipo cronobiológico, obtivemos predomínio de trabalhadores dos sexos feminino e masculino com cronótipo "moderadamente matutino", respectivamente $18,60 \%$ e $30,23 \%$.

Em relação às variáveis tempo na função e cronótipo, obtivemos predomínio de trabalhadores com maior tempo de trabalho com cronótipo "indiferente" (63,67 meses), seguidos pelo cronótipo "moderadamente matutino" (50,95 meses) e "definitivamente matutino" (48,50 meses).

Com relação à idade dos trabalhadores e ao cronótipo, os trabalhadores com média maior de idade apresentaram cronótipo "definitivamente vespertino". Em geral, as pessoas jovens tendem a ter características mais pronunciadas de vespertinidade e vão se tornando mais matutinas à medida que envelhecem (Monk et al., 1996; Reilly, Waterhouse \& Atkinson, 1997; Andrade, 1997). Steele et al. (1997), em sua pesquisa sobre as características de matutinidade e vespertinidade em residentes de medicina norte-americanos, verificaram que os indivíduos mais velhos tendiam a ser mais matutinos. Akerstedt e Torsvall (1981) afirmam que as pessoas vão se tornando mais matutinas ao longo dos anos.

As diferenças individuais constituem um aspecto fundamental na análise da adaptação de trabalhadores aos turnos a que estão submetidos (Menna-Barreto et al., 1993). Os cronótipos são importantes mecanismos cronobiológicos da estrutura temporal para determinar que o organismo é fisiologicamente diferente a cada momento do dia e, em conseqüência, tem, a cada momento, capacidade diferente de reagir aos estímulos ambientais, sejam eles físicos, químicos, biológicos ou sociais (Rotemberg, MennaBarreto \& Marques, 1997). Desta forma, as características de matutinidade/vespertinidade do trabalhador em turnos são um importante fator a ser levado em consideração na análise dos efeitos do trabalho em turnos sobre os trabalhadores.

Considerando o impacto do trabalho em turnos sobre a qualidade de vida e sobre a saúde dos trabalhadores, devemos considerar as características individuais, como idade, personalidade, tipo de ritmo (tendência à matutinidade/vespertinidade), assim como sua capacidade psicológica de adaptação, além de outros fatores, como a extensão da carga física ou intelectual do trabalho, a duração da jornada, o tipo de escala de turnos e as condições que cercam o local de trabalho, 
como os fatores climáticos, os níveis de ruído, o manuseio de matérias-primas especiais e produtos perigosos, etc. Também influem as condições sociais e domésticas em que vive o trabalhador, como a situação familiar, o número de filhos, bem-estar e aceitação do turno pela família (Rutenfranz, Knauth \& Fisher, 1989). Nesta pesquisa, procuramos contribuir com

\section{Conclusões}

Os resultados obtidos na presente pesquisa permitem as seguintes conclusões:

Houve uma distribuição homogênea com relação ao número de trabalhadores por turno de trabalho, o predomínio de trabalhadores do sexo masculino e o tempo na função em média de 4 anos e 6 meses.

Houve predomínio do cronótipo "moderadamente matutino" seguido do tipo "indiferente" e do tipo "definitivamente matutino". Encontramos apenas um trabalhador "definitivamente vespertino".

A maioria dos trabalhadores do turno matutino foi classificada como "moderadamente matutino" e "definitivamente matutino", demonstrando uma associação entre o cronótipo e o turno de trabalho.

A maioria dos trabalhadores do turno vespertino foi classificada como "mode- uma avaliação inicial, já que o setor da indústria química em que foi realizada a pesquisa atualmente conta com o turno noturno para atender à demanda da produção. Estudos complementares se fazem necessários, além da análise acerca das relações entre as características de matutinidade/vespertinidade e a produtividade.

radamente matutino”, o que pode acarretar um problema para estes trabalhadores.

Verificamos a presença tanto de trabalhadores do matutino como do vespertino com cronótipo "indiferente".

Houve predomínio de trabalhadores dos sexos feminino e masculino com cronótipo "moderadamente matutino".

Houve predomínio de trabalhadores com maior tempo na função com cronótipo "indiferente".

Os trabalhadores com média maior de idade apresentaram cronótipo "definitivamente vespertino”.

Nesta pesquisa não foram verificadas associações estatisticamente significativas entre o cronótipo e as características individuais.

\section{Agradecimentos}

À Comissão de Pesquisa - Estatística da Faculdade de Ciências Médicas da Universidade Estadual de Campinas, à Rita Geraldo Oshiro (Supervisora de Produção e Engenheira de Materiais) e à Marineide da Silva (Administradora de Empresas).

\section{Referências Bibliográficas}

ANDRADE, M. M. M. Padrões temporais das expressões da sonolência em adolescentes. 1997. Tese (Doutorado em Fisiologia Humana) Instituto de Ciências Biomédicas, Universidade de São Paulo.

BENEDITO-SILVA, A. A.; MENNA-BARRETO, L. S.; MARQUES, N.; TENREIRO, S. A selfassessment questionnaire for determination of morningness-eveningness types in Brazil. In: HAYES, D. K.; PAULY, J. E.; REITER, R. J. (ed.) Chronobiology: its role in clinical medicine, general biology and agriculture, part B. New York, 1990.

CAMPOS, M. L. P.; DE MARTINO, M. M. F. Caracterização do cronótipo das professoras de um colégio técnico de Campinas. Nursing, 2001.

CIPOLLA-NETO, J. Fisiologia do sistema de temporização circadiana. In: CIPOLLA-NETO,
J.; MARQUES, N.; MENNA-BARRETO, L. S. Introdução ao estudo da Cronobiologia. São Paulo: Ícone, 1988. p. 65-146.

DE MARTinO, M. M. F.; CEOLIM, M. F. Avaliação do cronótipo de enfermeiros de hospital universitário de ensino. Revista Ciências Médicas. 10(1):19-27. 2001.

DE MARTINO, M. M. F.; LING, S. Y. Características cronobiológicas de um grupo de alunos universitários de enfermagem. Revista Ciências Médicas. 13(1):43-49. 2004.

FISCHER, F. M. As demandas da sociedade atual: aspectos históricos do desenvolvimento do trabalho em turnos no mundo-conceitos, escalas de trabalho, legislação brasileira. In: FISCHER, F.M; MORENO, C. R. C.; ROTENBERG, L. Trabalho em turnos e noturno na sociedade 24 horas. São Paulo: Atheneu, 2003. p. 3-17. 
HORNE, J. Á.; ÖSTBERG, D. A self-assessment questionnaire to determine morningnesseveningness in human circadian rhythms. International Journal of Chronobiology. 4:97-110. 1976.

MENNA-BARRETO, L. S. et al. Individual differences in night and continuously-rotating shiftwork: seeking anticipatory rather than compensatory strategy. Ergonomics. 36:13540. 1993.

MENNA-BARRETO, L. S.; FORTUNATO, G. O que é Cronobiologia? In: CIPOLLA-NETO, J.; MARQUES, N.; MENNA-BARRETO, L.S. Introdução ao estudo da cronobiologia. São Paulo: Ícone; 1988. p. 15.

MONK, T. M. et al. Subjective alertness rhythms in elderly people. Journal of Biological Rhythms. 11(3):268-276. 1996.

REILLY, T.; WATERHOUSE, J.; ATKINSON, G. Aging, rhythms of physical performance, and adjustment to changes in the sleepactivity cycle. Journal of Occupational \&
Environmental Medicine. London, 54(11): 812-16. 1997.

REIMÃO, R. Sono. Revista Médica Roche. 10(9):16-19. 1988

ROTENBERG, L.; MENNA-BARRETO, L. S.; MARQUES, N. Desenvolvimento da Cronobiologia. In: MARQUES, N.; MENNABARRETO, L. S. (org). Cronobiologia: princípios e aplicações. São Paulo: Edusp, 1997. p. 17-21.

RUTENFRANZ, J.; KNAUTH. P.; FISCHER, F. M. Trabalho em turnos e noturno. São Paulo: Hucitec, 1989.

STEELE, M. T. et al. Morningness-eveningness preferences of emergency medicine residents are skewed toward eveningness. Acad. Emerg. Med. 4(7):699-705. 1997.

ZUBIOLI, M. A. S.; MIRANDA NETO, M. H.; SANT'ANA, D. M. G. Avaliação dos cronótipos dos auxiliares de enfermagem do Hospital Santa Casa de Paranavaí. PR. Arq. Ciênc. Saúde Unipar. 2(3):1-7. 1998. 